\title{
Gravitational wave experiments
}

\section{William O. Hamilton $\dagger^{1}$ and Ho Jung Paik}

† Department of Physics and Astronomy, Louisiana State University, Baton Rouge, LA 70803, USA

$\ddagger$ Department of Physics, University of Maryland, College Park, MD 20742, USA

\section{Introduction}

There were three oral sessions and one poster session for Workshop C1 on Gravitational Wave Experiments. There was also an informal experimental roundtable held one afternoon. The first two oral sessions were devoted mainly to progress reports from various interferometric and bar detector groups. A total of 15 papers were presented in these two sessions. The third session of Workshop $\mathrm{C} 1$ was devoted primarily to theoretical and experimental investigations associated with the proposed interferometric detectors. Ten papers were presented in this session. In addition, there were a total of 13 papers presented in the poster session. There was some overlap between the presentations in the third oral session and the posters since only two of the serious posters were devoted to technology not pertinent to interferometers.

In general, the papers showed the increasing maturity of the experimental aspects of the field since most presented the results of completed investigations rather than making promises of wonderful results some time in the future. Unfortunately, the limited time allotted to experimental reports made the session more like a session of contributed papers at a very large national meeting rather than a session where work could be presented and the merits of various approaches debated and discussed. Hopefully future GR meetings will have more time devoted to experimental aspects of General Relativity research.

\section{Sessions I and II}

The first paper in Session I was a report by Whitcomb on the status of the LIGO project. The five-year project received first-year funding in 1992 to begin design and construction of a two-facility observatory for the detection and study of gravitational waves from astrophysical sources. Sites have been selected (Hanford, Washington, and Livingston, Louisiana) and detailed design will begin soon. Under the present schedule, the facilities will be completed by 1997 and initial observations will begin in 1998. Abramovici followed

1 E-mail: PHMLTN@LSUVM (BITNET) or 7620::HAMILTON (SPAN) 
with a second paper which reported on the sensitivity of the LIGO $40 \mathrm{~m}$ prototype interferometer at Caltech. The lowest noise measured is $1.2 \times 10^{-18} \mathrm{~m} / \sqrt{\mathrm{Hz}}$ at $1 \mathrm{kHz}$ in displacement sensitivity. The noise spectrum is dominated by seismic noise below 120 $\mathrm{Hz}$ and by shot noise above $1.5 \mathrm{kHz}$.

After these two reports on the laser detectors, there were a series of reports on the progress of cryogenic bar detectors. First, there were reports on the detectors in operation. Hamilton discussed the ongoing experiment at LSU. The LSU 4 K detector has been in continuous operation since April 1991. Its noise level corresponds to an rms strain of $5 \times 10^{-19}$. This is the best noise level that anybody has ever reported, albeit by a small margin. The data is extraordinarily clean, showing fewer than 20 events per day which lie above the expected exponential distribution. He stressed the need for multiple detectors operating in conincidence, regardless of the sensitivity of individual detectors. Looking into the future, Hamilton discussed an interesting possibility of constructing a large $50 \mathrm{mK}$ sphere antenna. This idea is being studied jointly by the bar groups in the U.S. The sphere is omni-directional and may reach a sensitivity of $h<10^{-21}$ due to its large mass and multimode nature. Ricci discussed the experience obtained from long-term operation of the $4 \mathrm{~K}$ EXPLORER detector at CERN. After being upgraded, EXPLORER was in continuous operation from July 1990 until December 1991. Its noise temperature has been better than $10 \mathrm{mK}$ and the duty cycle of data taking larger than $70 \%$. The 6 months of coincidence data between the Rome and LSU detectors is still being analyzed.

Then there were reports on ongoing construction work for ultralow temperature bar detectors. Coccia reported the progress on the construction of the ultralow temperature detector NAUTILUS. A $2350 \mathrm{~kg} \mathrm{Al} 5056$ bar was successfully cooled to $95 \mathrm{mK}$ at CERN in 1991. After this cryogenic test, the detector was moved to Frascati in the spring of 1992. NAUTILUS is being reassembled and is expected to go into operation at the end of 1992. A progress report on a second ultralow temperature detector, being assembled at Stanford, was given by Mann. The cryostat has been extensively redesigned and modified to cool a $1800 \mathrm{~kg} \mathrm{Al} 5056$ antenna to $50 \mathrm{mK}$. Work is nearly complete on the Paik transducer and associated commercial dc SQUID for the first phase of the detector operation. This detector is expected to go into operation in the first half of 1993. The senstivity goal of both the Rome and Stanford ultralow temperature detectors is $\mathrm{h}=10^{-20}$.

Session I ended with Schutz's reassessment of the reported correlations between gravitational waves and neutrinos associated with SN1987a. He pointed out that one of the statistical tests used to establish the reported correlations between the room temperature gravitational wave antennas and the neutrino detectors is seriously flawed, and most other were devised a posteriori and contain considerable freedom to make choices that strengthen the correlations. Schutz concludes that the claimed gravitational wave-neutrino correlations are likely to be due, not to any physical effects, but simply to chance. There was disagreement expressed during the discussion but it was decided to postpone the argument until everything had been published.

Session II started with Blair and Tobar's progress report on the niobium bar antenna at Perth. The highest $Q$ achieved with the niobium antenna at $4 \mathrm{~K}$ is $2.3 \times 10^{8}$. The vibration isolation system for the Perth antenna has been completely rebuilt and the detector is undergoing reassembly. Blair then discussed the status of the AIGO project. It is undergoing a second review in Australia following a previous recommendation by 
ASTEC that the observatory be $50 \%$ Australian funded if international funding could be secured for the balance. Nicholson presented a coincidence analysis between the two prototype interferometric detectors at Glasgow and the Max Planck Institute in Garching. The 100 hours of coincidence data was used to test automatic data analysis algorithms which search for signals of astrophysical origin. Progress on the intermediate size laser interferometer in Japan was reported by Kawashima. The construction of TENKO-100, a $100 \mathrm{~m} 100$ bounce delayline interferometer, will be completed by the end of the summer of 1992. The hard work of attaining high sensitivity will follow.

Attention was then shifted again to real data. Astone presented a new upper limit on gravitational waves obtained from the long-term operation of EXPLORER. The detector noise temperature was $10 \mathrm{mK}$ and corresponded to $\mathrm{h}=6 \times 10^{-19}$ for short bursts of gravitational waves. The new limit ( less than 0.1 events per day for bursts with $h$ larger than $4 \times 10^{-17}$ ) represents an improvement by an order of magnitude over the previous limit obtained with the Stanford detector in 1982. In the next paper, Bertotti described the search for low-frequency gravitational waves from the tracking data for the interplanetary spacecraft ULYSSES. The data was taken in a four-week period starting February 20, 1992 but unfortunately the data analysis has not been completed.

The final two papers in Session II were on improvements on the laser interferometers. Newton reported some improvements to the Glasgow prototype interferometer. There was a lengthy discussion about the effects of relatively poor vacuum on the performance of mirrors. Drever then discussed an idea of the late Brian Meers, an "ultrasensitive configuration" for laser interferometers, using a double sideband recycling technique.

\section{Roundtable}

An informal experimental roundtable was held during one afternoon. The intent was to allow discussion, unconstrained by formal structure, about the experimental problems and the future direction of the field. One question, posed by Blair, occupied much of the discussion. Blair asked: "If we were to start anew with bar detectors, for what frequency should we build them?" Thorne answered immediately that they should be designed for the lowest possible frequency, whereupon Will suggested that $1.4 \mathrm{kHz}$ would be a good place. Considerable discussion ensued with the final advice to the experimentalists being that no one really knows the answer so they should build what they can do best. We all will then wait to see what is found.

Pizzella also announced, for the Rome and LSU groups, that the first result of the joint coincidence analysis is that for the 6 months studied there were no coincidences with an energy greater than $200 \mathrm{mK}$. This corresponds to $\mathrm{h}=3 \times 10^{-18}$.

\section{Session III}

The first paper in Session III was presented by Cutler for the Caltech relativity group. He presented their estimates for the frequencies and the duration for the inspiraling of coalescing neutron star binaries. They have also estimated the parameters for neutron star-black hole and black hole-black hole binaries. Monte Carlo simulations show that 
if such signals are detected, using undescribed pattern matching techniques, that the signal to noise ratio will be relatively high, enabling the masses and the distances of the sources to be measured. Lobo then presented a paper with Krolak and Meers which set a formidable technological challenge, showing that if an interferometer were to be run using dual recycling and if the recycling tuning could be dynamically tuned as the signal from the binary was detected, the signal to noise would be greatly improved. How such detection and dynamic tuning could be accomplished was left as an exercise for the audience. Krolak then discussed the reverse problem: How well can one determine the mass, range, and phase of a detected binary system? He used a network of 3 interferometers, the two LIGO detectors in the US and the VIRGO detector near Pisa. The results show that range is the most uncertain of the parameters, but the signals may be detectable at ranges as large as $200 \mathrm{Mpc}$.

Bender discussed the possibility of massive black holes at the center of galaxies and the gravitational radiation signature from neutron stars spiraling into such objects. Such signals are inaccessible to anything but a space-based system.

Passive and active seismic isolation systems occupied the remainder of the session. Several of the papers on seismic isolators seemed to revisit much of the territory previously explored by those designing resonant bar detectors. Shoemaker discussed the design, at MIT, of a passive seismic isolator for LIGO. He pointed out the limits to the performance of such isolators caused by internal resonances in the components of the isolation stack. Gonzalez discussed the work for LIGO of the Syracuse group and the new theoretical approach she has used to investigate the old problem of thermal noise in a pendulum. Her work does not directly involve a normal mode expansion and seems to give reasonable results. Saulson continued with a discussion of the effects, on a LIGO pendulum isolator, of the stored tensile energy in highly stressed wires. Since the stored energy is so large, he asked if its release could be a noise source which is much larger than the thermal noise. This is a problem which still doesn't have an answer, either theoretical or experimental. The question centers about whether the energy is released all at once or in small increments. Barone gave the results of a computer program designed to optimize the performance of a static steel and rubber stack and pendulum support. Its optimization criteria led to successively smaller masses as one goes up the stack.

Newell gave a progress report on the Colorado program to develop an active isolation system for the mirrors of a LIGO system. As with all experiments, the meeting came at an inconvenient time, and he had not been able to close all of the feedback loops before he had to leave to report on his results. The vertical motion loops had been closed,however, and the prototype system performed as expected. Notcutt then spoke about the seismic isolators at Perth which eliminate steel and rubber stacks and replace them with star shaped plates in flexure. Magnets are used on the plates to provide damping. These have been already installed in the redesigned Perth resonant bar detector which is set for a cryogenic run Real Soon Now and are also being used in the Perth laboratory interferometer prototype. Their isolation seems to be quite good and isolators of this type should also perform well if cooled.

\section{Conclusions}

With the emergence of the long-baseline laser interferometers, the number of papers presented in the Gravitational Wave Experiments Workshop has exploded. The future 
of gravitational wave astronomy looks bright. The construction of ultralow temperature bar detectors is nearly complete. The LIGO project is moving ahead with the design and construction of two $4 \mathrm{~km}$ baseline interferometers in the U.S. and the VIRGO group will build at least one in Europe. These sensitive antennas should be operational by the turn of the century. By that time there may even be a few very massive sphere antennas carrying out an all-sky search for gravitational waves in coincidence with the laser interferometers.

It appears that another decade of hard work is ahead of us. But the payoff may not be that far away. The new detectors are now beginning to reach very interesting sensitivity levels. If Nature cooperates, who knows? One of us may be reporting a clear detection of gravitational waves before the turn of the century, perhaps at one of these GRG meetings. 
